\title{
A Novel Modelling Method for Fault Detection and Diagnosis in Large Water Chillers without Water Flow Measurements
}

\author{
C.Y. Zheng \\ School of Marine Engineering \\ Jimei University \\ Xiamen 361021, China \\ Fujian Provincial Key Laboratory of Naval Architecture and \\ Ocean Engineering \\ Xiamen 361021, China
}

\author{
W. Chen* \\ School of Marine Engineering \\ Jimei University \\ Xiamen 361021, China \\ Fujian Provincial Key Laboratory of Naval Architecture and \\ Ocean Engineering \\ Xiamen 361021, China \\ *Corresponding author
}

\begin{abstract}
Conventional fault detection and diagnosis in water chillers needs water flow measurements to calculate system load. However, the measurement of water flow meters usually fluctuates greatly, and high accuracy flow meters are very expensive. In this paper, a novel modelling method using a new reference model for fault detection and diagnosis in water chillers was developed, and the real evaporator water flow rate was replaced by a pseudo evaporator water flow rate that was equal to the designed evaporator water flow rate or the average evaporator flow rate under stable operation conditions. The newdeveloped reference model was validated by the data sets from ASHRAE 1043-RP. The validation results showed that the new developed reference model has good prediction accuracy and clear diagnosis rules, which can be used for fault detection and diagnosis in water chillers in the absence of water flow measurements.
\end{abstract} model

Keywords-fault detection and diagnosis; water chiller; reference

\section{INTRODUCTION}

Fault detection and diagnosis (FDD) has been an important element in the field of heating, ventilating, air-conditioning and refrigeration (HVAC\&R) since the late 1980s, which much lagged behind the FDD in critical systems such as aerospace exploration, nuclear power plants and chemical process plants. The main cause is that the consequences of faults in building HVAC\&R are comfort-related, environmental and economic instead of safety-critical [1]. There is less possibility to build an expensive FDD system for HVAC \& R systems because the benefits of FDD for them are much lower than that for critical systems. With the rapid development of electronic and computer technology, the gradual reduction of hardware cost, the increasing complexity of building HVAC\&R system and the increasing power consumption, more and more building owners introduce FDD system into their building HVAC\&R systems so as to save energy, reduce operation cost, improve indoor air quality and reduce customers complaints.
Most modern large building air-conditioning systems adopt water chillers, in tropical and subtropical area such as Hong Kong, water chillers consume $35 \sim 40 \%$ of the total building power consumption [2], thus the fault detection and diagnosis of water chiller received extensive attention [2-14].

In the research on FDD of water chillers, clear firstprinciple theory, abundant empirical knowledge and tremendous amount of operating data from building management system (BMS) can help researchers develop a lot of data-driven FDD models, e.g. simple linear regression model [3], Bi-quadratic regression model [3], multivariate polynomial regression model [2,4-9], Gordon-Ng simplified model [10], Gordon-Ng universal model [11,12], Lee's simplified model [13] and artificial neural networks model [14]. Most of these models use system cooling load as one of their inputs. Since the calculation of system cooling load needs water flow rate, the system needs to install water flow meters. However, the measurements of water flow rate usually fluctuate in a relatively large range, which will have a bad impact on the FDD of water chillers, and high accuracy water flow meter is very expensive. To install high accuracy flow meters can not meet low-cost requirements for the FDD of HVAC\&R system. Therefore, the study on FDD in water chillers without water flow measurements is significant.

\section{TYPICAL WATER CHILLER AND ITS MEASUREMENTS}

Most large building air-conditioning systems adopt water chiller with constant evaporator water flow rate and condenser water flow rate. The configuration of a typical water chiller is as shown in Figure 1. The chiller consists of three loops, e.g. refrigerant loop, evaporator water loop and condenser water loop. The chiller's refrigerant loop couples with the evaporator water loop and the condenser water loop through evaporator and condenser respectively. In order to facilitate control and management, most commercial chiller systems measure some important data including evaporator water flow rate $\left(\mathrm{M}_{\mathrm{evw}}\right)$, the entering and leaving temperatures of evaporator water(TEI\&TEO), condensing water flow rate $\left(\mathrm{M}_{\mathrm{cdw}}\right)$, the entering and leaving temperatures of condensing 
water(TCI\&TCO), evaporating temperature $\left(\mathrm{P}_{\mathrm{ev}}\right)$, condensing temperature $\left(\mathrm{P}_{\mathrm{cd}}\right)$, compressor suction and discharge temperature of refrigerant $\left(\mathrm{T}_{\text {suc }} \& \mathrm{~T}_{\mathrm{dis}}\right)$, compressor lube oil temperature $\left(\mathrm{T}_{\mathrm{oil}}\right)$ and electrical signal of compressor. Some chiller systems also measure the temperature of liquid line (TL) for FDD and management.

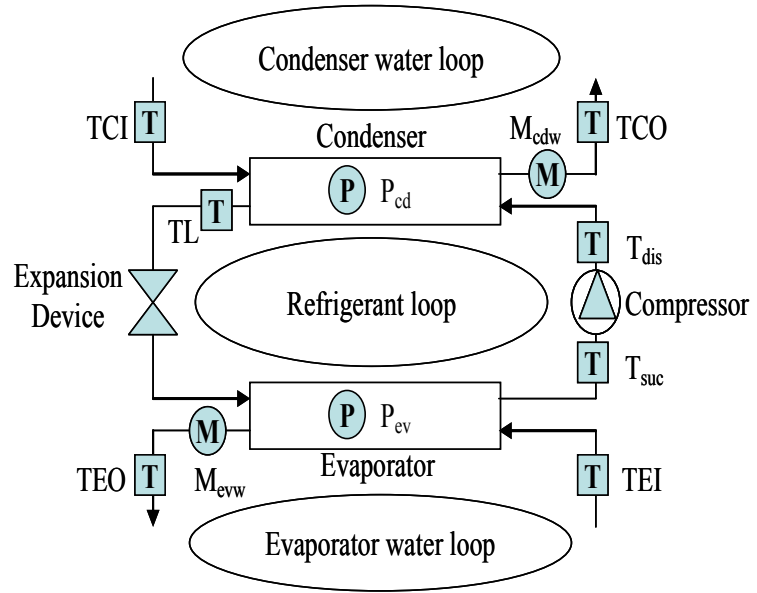

FIGURE I .CONFIGURATION OF A TYPICAL CHILLER.

\section{NEW REFERENCE MODEL AND ITS VALIDATION}

Since the building owners' investment on building airconditioning system is different, the information that can be obtained is usually different. Therefore, it is necessary to study different system models so as to adapt to the requirements of FDD under different conditions. From cost-saving point of view, a FDD model which uses low-cost measurements should be studied. Literature review showed that a multivariate polynomial regression model has been widely used in the FDD of water chillers [2, 4-9]. The main reason is that it has good prediction accuracy and easy to be trained. Its detailed formation is shown in equation (1) (named reference model 1).

$$
\begin{aligned}
& Y_{1}=f\left(Q_{e v}, T E O, T C I\right)+\varepsilon \\
& =a_{0}+a_{1} Q_{e v}+a_{2} T E O+a_{3} T C I+a_{4} Q_{e v} \times T E O+a_{5} Q_{e v} \times T C I \\
& +a_{6} T E O \times T C I+a_{7} Q_{e v}{ }^{2}+\varepsilon \\
& \text { or } \\
& Y_{1}=a_{0}+a_{1} Q_{e v}+a_{2} T E O+a_{3} T C I+a_{4} Q_{e v} \times T E O+a_{5} Q_{e v} \times T C I \\
& +a_{6} T E O \times T C I+a_{7} Q_{e v}{ }^{2}+a_{8} T E O^{2}+a_{9} T C I^{2}+\varepsilon
\end{aligned}
$$

Where, the error term is $\varepsilon \sim N\left(0, \sigma^{2}\right)$.The inputs are cooling load $(\mathrm{Qev})$, evaporator water leaving temperature (TEO) and condenser water entering temperature (TCI). The outputs $Y$ are necessary parameters from measurement or derived from measurement.

\section{A. Formation Of New Reference Model}

When water flow measurement is not available, the system cooling load can not be obtained directly. In this condition, pseudo cooling load can be used to replace real chiller load. Pseudo chiller load can be calculated by pseudo water flow rate which can be obtained by the following two ways:

(1) In a well-designed water chiller system, the real water flow rate is always very close to its designed flow rate.
Therefore, pseudo water flow rate is equal to designed water flow rate;

(2) All large building air-conditioning systems are generally required to go through a trial operation stage before they are officially put into use. In this stage, non-contact type flow sensor such as ultrasonic flow meter can be rented so as to obtain real water flow measurements in the stable operating condition. The measured average water flow information can be regarded as the system designed water flow rate. Therefore, pseudo water flow rate is regarded to be equal to the average flow rate.

The new reference model with pseudo chiller load is expressed as equation (2):

$$
\begin{aligned}
& Y_{2}=f\left(Q_{e v}^{*}, T E O, T C I\right)+\varepsilon \\
& =a_{0}+a_{1} Q_{e v}^{*}+a_{2} T E O+a_{3} T C I+a_{4} Q_{e v}^{*} \times T E O+a_{5} Q_{e v}^{*} \times T C I \\
& +a_{6} T E O \times T C I+a_{7} Q_{e v}^{* 2}+\varepsilon
\end{aligned}
$$

Where, $Q_{e v}{ }^{*}$ is pseudo chiller load and it can be calculated by equation (3):

$Q_{e v}^{*}=C_{p w} \times M_{e v w_{-} d e s i g n} \times(T E I-T E O)$ or $Q_{e v}^{*}=C_{p w} \times M_{e v w_{-} \text {rating }}(T E I-T E O)$

Where, $C_{p w}$ is specific heat of water, $M_{\text {evw_esign }}$ is designed evaporator water flow, $M_{\text {evw_rating }}$ is the stable evaporator water flow obtained in trial operation stage.

\section{B. Validation}

1) Data sets from ASHRAE 1043-RP: In order to validate whether the new-developed reference model has good prediction accuracy and clear diagnosis rules or not, the data sets from ASHRAE 1043-RP are introduced. ASHRAE research project 1043-RP was sponsored by ASHRAE. It conducted experimental studies to produce both fault-free and faulty data sets to develop and evaluate FDD methods. The system cooling load ranged from $25 \%$ to $100 \%$ of the cooling capacity. Eight typical chiller faults including reduced evaporator water flow (RedEvW), reduced condenser water flow (RedCdW), excess oil (ExOil), refrigerant overcharge (RefOver), refrigerant leakage (RefLeak), condenser fouling (CdFoul), non-condenserable gas $(\mathrm{Ncg})$ and defective pilot valve (DefPVal) were monitored. All faults except defective pilot valve were tested from fault severity level 1 to level 4 , the detailed information is shown in Table 1. 
TABLE I .SEVEN TYPICAL CHILLER FAULTS AND THEIR SIMULATION METHODS IN ASHRAE 1043-RP.

\begin{tabular}{|c|c|c|c|c|c|c|}
\hline Faults & $\begin{array}{l}\text { Simulation } \\
\text { method }\end{array}$ & $\begin{array}{l}\text { Normal } \\
\text { operation }\end{array}$ & Level 1 & Level 2 & Level 3 & Level 4 \\
\hline RedEvW & $\begin{array}{l}\text { Reduce } \\
\text { water flow }\end{array}$ & $13.6 \mathrm{~kg} / \mathrm{s}$ & $10 \%$ & $20 \%$ & $30 \%$ & $40 \%$ \\
\hline RedCdW & $\begin{array}{l}\text { Reduce } \\
\text { water flow }\end{array}$ & $17.0 \mathrm{~kg} / \mathrm{s}$ & $10 \%$ & $20 \%$ & $30 \%$ & $40 \%$ \\
\hline ExOil & Add oil & $10 \mathrm{~kg}$ & $14 \%$ & $32 \%$ & $50 \%$ & $68 \%$ \\
\hline RefOver & $\begin{array}{l}\text { Add } \\
\text { refrigerant }\end{array}$ & $136 \mathrm{~kg}$ & $10 \%$ & $20 \%$ & $30 \%$ & $40 \%$ \\
\hline RefLeak & $\begin{array}{l}\text { Reduce } \\
\text { refrigerant }\end{array}$ & $136 \mathrm{~kg}$ & $10 \%$ & $20 \%$ & $30 \%$ & $40 \%$ \\
\hline CdFoul & Block tube & 164 tubes & $\begin{array}{l}20 \text { tubes } \\
12 \%\end{array}$ & $\begin{array}{ll}s 33 & \text { tube } \\
20 \% & \end{array}$ & $\begin{array}{l}\text { s49 tubes } \\
30 \%\end{array}$ & $\begin{array}{l}\text { s } 74 \text { tubes } \\
45 \%\end{array}$ \\
\hline Ncg & $\begin{array}{l}\text { Add } \\
\text { nitrogen }\end{array}$ & $\begin{array}{l}\text { No } \\
\text { nitrogen }\end{array}$ & $0.0454 \mathrm{~kg}$ & $0.0726 \mathrm{~kg}$ & $0.10 \mathrm{~kg}$ & $0.245 \mathrm{~kg}$ \\
\hline
\end{tabular}

2) Prediction accuracy of new reference model: For FDD, whether a system model can predict the system's fault-free operating parameters with high accuracy or not is one of the main criteria to determine its value. In order to validate new reference model's prediction accuracy, the normal operating data set "Normal 2" of ASHRAE 1043-RP is used to train the new reference model, and data set "Normal 1" is used to further validate the new reference model's prediction accuracy

The coefficient of determination (R2) of new reference model is shown in Table 2. All R-square values are larger than $95 \%$, which indicates the very strong goodness-of-fit of the regression models and good prediction accuracy.

TABLE II .COEFFICIENT OF DETERMINATION OF NEW REFERENCE MODEL.

\begin{tabular}{llll}
\hline Parameters & $\begin{array}{l}\text { R-square } \\
\text { Normal 2 }\end{array}$ & (\%) & $\begin{array}{l}\text { R-square } \\
\text { Normal 1 }\end{array}$ \\
\hline TEI-TEO & 99.9951 & 99.9923 \\
TCO-TCI & 99.9788 & 99.9483 \\
$\mathrm{~T}_{\text {ev }}$ & 99.2182 & 97.0089 \\
$\mathrm{~T}_{\text {suc }}$ & 99.9084 & 99.8159 \\
$\mathrm{~T}_{\text {cd }}$ & 99.9478 & 99.7192 \\
$\mathrm{TL}$ & 99.9449 & 99.9071 \\
$\mathrm{~T}_{\text {dis }}$ & 99.4803 & 99.4446 \\
$\mathrm{~T}_{\text {oil }}$ & 97.1484 & 96.1856 \\
\hline
\end{tabular}

3) Fault diagnosis rules of new reference model: In order to study the impact on fault diagnosis caused by the new reference, the faulty data sets of ASHRAE 1043-RP in fault severity level 4 are used to carry out trend analysis for diagnosis rules extraction, and compared them with the diagnosis rules of the reference model 1 . The threshold of trend analysis is three times standard deviations of the trained reference model. The diagnosis rules of reference new reference model and model 1 are shown in Table 3 and 4 .

From Table 3 and 4, it can be found that all six faults have the same diagnosis rules except reduced evaporator water. The main reason is that reference model 1 and new reference model are essentially the same when evaporator water has not fault. When reduced evaporator water occurs, the cooling load of new reference model is calculated as pseudo cooling load, which is larger than real cooling load. Thus predicted compressor power consumption and condenser load of new reference model are larger than real ones. Moreover, larger predicted compressor power consumption results in larger real coefficient of performance (COP); and larger predicted condenser load causes lower real condensing pressure, liquid line temperature, liquid line subcooling (TL_sub) and condenser water temperature difference. In addition, from diagnosis rules in Table 3, reduced evaporator water fault can be diagnosed by decreased condenser water temperature difference. Furthermore, the calculation results show that condenser water temperature difference is very sensitive to reduced evaporator water, even in fault severity $1,10 \%$ evaporator water reduced. The residual between real measurement and predicted value locates -1.897 -11.179 times threshold, and mean residual is -6.080 times threshold. Consequently, reduced evaporator water can be diagnosed in fault severity level 1 or lower level. 
TABLE III: Diagnosis rules of new reference model.

\begin{tabular}{lcccccccccc}
\hline $\begin{array}{l}\text { Rarameters } \\
\text { Faults }\end{array}$ & $\mathrm{T}_{\mathrm{ev}}$ & $\mathrm{T}_{\mathrm{cd}}$ & $\mathrm{T}_{\mathrm{suc}}$ & $\mathrm{T}_{\text {dis }}$ & $\mathrm{TL}$ & $\mathrm{TL} \_$sub & $\begin{array}{l}\text { TEI- } \\
\text { TEO }\end{array}$ & $\begin{array}{l}\mathrm{TCO}- \\
\mathrm{TCI}\end{array}$ & $\mathrm{KW}$ & $\mathrm{COP}$ \\
\hline RedEvW & $\bullet$ & $\downarrow$ & $\bullet$ & $\bullet$ & $\downarrow$ & $\downarrow$ & $\bullet$ & $\downarrow$ & $\downarrow$ & $\uparrow$ \\
RedCdW & $\uparrow$ & $\uparrow$ & $\bullet$ & $\uparrow$ & $\uparrow$ & $\uparrow$ & $\bullet$ & $\uparrow$ & $\uparrow$ & $\downarrow$ \\
ExOil & $\bullet$ & $\bullet$ & $\bullet$ & $\bullet$ & $\bullet$ & $\bullet$ & $\bullet$ & $\bullet$ & $\uparrow$ & $\downarrow$ \\
RefOver & $\bullet$ & $\uparrow$ & $\bullet$ & $\uparrow$ & $\downarrow$ & $\uparrow$ & $\bullet$ & $\uparrow$ & $\uparrow$ & $\downarrow$ \\
RefLeak & $\bullet$ & $\downarrow$ & $\bullet$ & $\downarrow$ & $\uparrow$ & $\downarrow$ & $\bullet$ & $\bullet$ & $\bullet$ & $\bullet$ \\
CdFoul & $\bullet$ & $\uparrow$ & $\bullet$ & $\uparrow$ & $\uparrow$ & $\bullet$ & $\bullet$ & $\uparrow$ & $\uparrow$ & $\downarrow$ \\
Ncg & $\bullet$ & $\uparrow$ & $\bullet$ & $\uparrow$ & $\bullet$ & $\uparrow$ & $\bullet$ & $\uparrow$ & $\uparrow$ & $\downarrow$ \\
\hline
\end{tabular}

Note: $\uparrow$ indicates an increasing trend when fault occurs; $\downarrow$ indicates a decreasing trend when fault occurs;

indicates no discernible trend when fault occurs.

TABLE IV. DIAGNOSIS RULES OF REFERENCE MODEL 1.

\begin{tabular}{|c|c|c|c|c|c|c|c|c|c|c|}
\hline $\begin{array}{l}\text { Parameters } \\
\text { Faults }\end{array}$ & $\mathrm{T}_{\mathrm{ev}}$ & $\mathrm{T}_{\mathrm{cd}}$ & $\mathrm{T}_{\text {suc }}$ & $\mathrm{T}_{\mathrm{dis}}$ & $\mathrm{TL}$ & $\begin{array}{l}\mathrm{TL}_{-} \\
\text {sub }\end{array}$ & $\begin{array}{l}\text { TEI- } \\
\text { TEO }\end{array}$ & $\begin{array}{l}\text { TCO } \\
-\mathrm{TCI}\end{array}$ & KW & $\mathrm{COP}$ \\
\hline RedEvW & $\bullet$ & $\bullet$ & $\downarrow$ & $\bullet$ & $\bullet$ & $\bullet$ & $\uparrow$ & $\bullet$ & $\bullet$ & $\bullet$ \\
\hline RedCdW & $\uparrow$ & $\uparrow$ & $\bullet$ & $\uparrow$ & $\uparrow$ & $\uparrow$ & $\bullet$ & $\uparrow$ & $\uparrow$ & $\downarrow$ \\
\hline ExOil & $\bullet$ & $\bullet$ & $\bullet$ & $\bullet$ & $\bullet$ & $\bullet$ & $\bullet$ & $\bullet$ & $\uparrow$ & $\downarrow$ \\
\hline RefOver & $\bullet$ & $\uparrow$ & $\bullet$ & $\uparrow$ & $\downarrow$ & $\uparrow$ & $\bullet$ & $\uparrow$ & $\uparrow$ & $\downarrow$ \\
\hline RefLeak & $\bullet$ & $\downarrow$ & $\bullet$ & $\downarrow$ & $\uparrow$ & $\downarrow$ & $\bullet$ & $\bullet$ & $\bullet$ & $\bullet$ \\
\hline CdFoul & $\bullet$ & $\uparrow$ & $\bullet$ & $\uparrow$ & $\uparrow$ & $\bullet$ & $\bullet$ & $\uparrow$ & $\uparrow$ & $\downarrow$ \\
\hline $\mathrm{Ncg}$ & $\bullet$ & $\uparrow$ & $\bullet$ & $\uparrow$ & $\bullet$ & $\uparrow$ & $\bullet$ & $\uparrow$ & $\uparrow$ & $\downarrow$ \\
\hline
\end{tabular}

\section{CONCLUSIONS}

A novel modeling method with a new reference model for FDD in water chillers where the water flow measurements are unreliable or unavailable was developed. Pseudo evaporate water flow rate obtained from design parameters or average evaporator flow rate in trial operation stage is used to calculate pseudo cooling load. The effectiveness of new reference model is validated by both fault-free and faulty data sets from ASHRAE 1043-RP. Good prediction accuracy, clear diagnosis rules and the ability to diagnose reduced evaporator water flow fault in low fault severity level indicate that the new reference model can be considered as an alternative choice for modelbased FDD in chillers.

\section{ACKNOWLEDGEMENTS}

The support from Natural Science Foundation Project of Fujian Province (2013J01201), Planning Project of Science and Technology Department of Fujian Province (201205180008), \& Science and Technology Project of Education Department of Fujian Province (JA12187) are acknowledged.

\section{REFERENCES}

[1] Braun, J.E., Automated fault detection and diagnosis for vapor compression equipment. Journal of Solar Energy Engineering, 125, pp. 266-274, 2003.

[2] Cui, J.T., \& Wang, S.W., A model-based online fault detection and diagnosis strategy for centrifugal chiller systems. International Journal of Thermal Sciences, 44, pp. 986-999, 2005.
[3] Swider, D.J., A comparison of empirically based steady-state models for vapor compression liquid chillers. Applied Thermal Engineering, 23(5), pp. 539-56, 2003.

[4] DOE (Department of Energy). DOE 2 reference manual, part 1, version 2.1. Berkeley (California): Lawrence Berkeley National Laboratories, 1980.

[5] Comstock, M.C., Braun, J.E., \& Groll, E.A., The sensitivity of chiller performance to common faults. HVAC\&R Research, 7(3), pp. 263279, 2001.

[6] Jia, Y.Z., \& Reddy, T.A., Characteristic physical parameter approach to modeling chillers suitable for fault detection, diagnosis, and evaluation. Journal of Solar Energy Engineering, (125), pp. 258-265, 2003.

[7] Reddy, T.A., Development and Evaluation of Simple Model-based Automated Fault Detection and Diagnosis (FDD) Method Suitable for Process Faults of Large chillers. ASHRAE Transactions, 113(2), pp. 27-39, 2007

[8] Zhou, Q., Wang, S.W., \& Xiao, F., A novel strategy for the fault detection and diagnosis of centrifugal chiller systems. HVAC \& R Research, 15(1), pp. 57-75, 2009.

[9] Xiao, F., Zheng, C.Y., \& Wang S.W., A fault detection and diagnosis strategy with enhanced sensitivity for centrifugal chillers. Applied Thermal Engineering, 31, pp. 3963-3970, 2011.

[10] Gordon J.M., Ng KC, Chua HT. Centrifugal chillers: thermodynamic modelling and a diagnostic case study. International Journal of Refrigeration, 18(4), pp. 253-7, 1995.

[11] Ng, K.C., Chua, H.T., Ong W., Lee, S.S., \& Gordon, J.M., Diagnostics and optimization of reciprocating chillers: theory and experiment. Applied Thermal Engineering, 17(3), pp. 263-76, 1996.

[12] Gordon, J.M., Ng, K.C., Cool thermodynamics. Cambridge (UK): Cambridge International Science Publishing, 2000.

[13] Lee T.S. Thermodynamic modelling and experimental validation of screw liquid chillers. ASHRAE Transactions, 110(1), pp. 206-16, 2004. 
[14] Swider, D.J., Browne, M.W., Bansal, P.K., \& Kecman, V., Modelling of vapor-compression liquid chillers with neural networks. Applied Thermal Engineering, 21 (3), pp. 311-329, 2001. 